\title{
063 ADDRESSING HIDDEN BARRIERS TO INSTITUTIONAL DELIVERIES - A KEY INTERVENTION FOR REDUCING MATERNAL MORTALITY IN RURAL ZAMBIA
}

Victor M Mukonka, ${ }^{1}$ Fionnuala M McAuliffe, ${ }^{2}$ Sarai B Malumo, ${ }^{3}$ Cephas C Sialubanje, ${ }^{4}$ Patricia E Fitzpatrick ${ }^{5}$. ${ }^{1}$ Department of Public Health, Copperbelt University, School of Medicine, Ndola, Zambia; School of Public Health, Physiotherapy \& Population Science, University College Dublin, Dublin, Ireland; ${ }^{2}$ UCD Obstetrics \& Gynaecology, School of Medicine \& Medical Sciences, UCD, Dublin, Ireland; ${ }^{3}$ Country Office, World Health Organisation, Lusaka, Zambia; ${ }^{4}$ Monze District Health Office, Ministry of Health, Monze, Zambia; ${ }^{5}$ School of Public Health, Physiotherapy \& Population Science, University College Dublin, Dublin, Ireland

\subsection{6/bmjopen-2015-forum2015abstracts.63}

Background In Zambia, majority rural women deliver at home without skilled birth attendants, leading to high maternal mortality rate (MMR) 591/100,000 live births; among highest worldwide $60 \%$ Zambians live below poverty line. Institutional delivery by skilled birth attendants is considered most important strategy to reduce MMR. Institutional deliveries are low (48\%) despite high single antenatal attendance (93\%). An important barrier to institutional delivery identified was demands by health providers requesting women to bring delivery supplies and mother/baby clothes.

Objectives The aim of this study was to determine the effect of provision of non-financial incentives on institutional deliveries in rural Monze district, Zambia.

Methods A one-year community intervention trial was conducted (Jan-Dec 2014) supported by UNICEF and WHO. Two comparable rural regions in Monze separated by central urban region, intervention arm expectant women who chose delivering at health facility received a mother-baby delivery-pack at delivery containing basic delivery supplies as non-financial incentives, control arm continued with routine health services. Primary outcome-measure was comparison of the number of institutional deliveries in the two arms over one year, and comparing institutional deliveries before (2012 \& 2013) and after (2014) the intervention.

Result There was a 43\% increase in institutional deliveries in the intervention arm in $2014(n=2396)$ compared to $2013(n=1674)$, $2012(\mathrm{n}=1680) ; \mathrm{p}<0.000$, while in the control arm the numbers of deliveries did not significantly change over the three years (2012 ( $\mathrm{n}=1182) ; 2013(\mathrm{n}=1322) ; 2014(\mathrm{n}=1182) ; \mathrm{p}>0.103)$. Conclusion The mother-baby delivery pack provides highimpact, low-cost, easier-to-replicate and scale-up intervention using existing health systems. The pack was developed responding to hidden barriers identified by end users in the community through cross-sectional survey conducted prior to the study. The results provide scientific evidence for policymakers to design effective interventions to overcome reversible barriers hindering facility utilisation by pregnant-women, a key intervention for reducing MMR. 